\title{
Hassas mutlak nokta konumlamada GIPSY-OASIS II v6.4 yazılımı ile elde edilen varyans kovaryans matrisinin güvenirliğinin araştırılması
}

\author{
Bahattin Erdoğan ${ }^{1 *}$ (D), Orhan Kayacık ${ }^{1}$ (D), Ali Hasan Doğan ${ }^{1}$ (D) \\ ${ }^{1}$ Yıldız Teknik Üniversitesi, Davutpaşa Kampüsü, Inşaat Fakültesi, Harita Mühendisliği Bölümü, Esenler, İstanbul.
}

Öz: Hassas Mutlak Konum Belirleme (Precise Point Positioning - PPP) tekniği, harita/geomatik mühendisliği uygulamalarında son yıllarda öne çıkan önemli konulardan birisidir. PPP tekniğinde konumu belirlenecek noktada yalnızca bir tek Küresel Navigasyon Uydu Sistemleri (Global Navigation Satellite Systems (GNSS)) alıcısının kullanılması yeterlidir. Bu nedenle kullanıcılar bu tekniği birçok uygulamada geleneksel bağll konumlama yöntemine göre tercih etmektedirler. PPP tekniği ile yapılan çalışmalarda veri değerlendirme ve analiz aşamalarında günümüzde genellikle bilimsel yazılımlar kullanılmaktadır. Bu yazılımlardan elde edilen Varyans kovaryans (VKV) matrisleri ise çok iyimser sonuçlar vermektedir. VKV matrislerinden hesaplanan değerler istatistiksel yorumlamalarda önemli etkiye sahip unsurlardır. GNSS veri değerlendirmesi sonucunda elde edilen VKV matrisleri deformasyon analizi gibi jeodezik çalışmalarda kullanılmaktadır. Bu bağlamda araştırmacılar VKV matrislerini ölçekleme ihtiyacı hissetmektedirler. Bu çalışmada, Uluslararası GNSS Servis (International GNSS Service - IGS) ağına ait 10 adet sürekli gözlem yapan sabit referans istasyonlarının, 2014 yllına ait 11 günlük verileri GIPSY-OASIS II v6.4 bilimsel yazllımı kullanılarak PPP tekniği ile analiz edilmiştir. 2, 4, 6, 8, 12 ve 24 saatlik gözlem süreleri için GIPSY-OASIS II v6.4 PPP sonuçlarından elde edilen VKV matrislerine ait ölçek faktörü kestirilmesi amaçlanmıştır. Analiz sonuçlarına göre ölçek faktörü değerlerinin gözlem süresi arttıkça büyüdüğ̈̈ gözlenmiştir.

Anahtar Sözcükler: Hassas mutlak konum belirleme, GIPSY-OASIS II v6.4, Varyans kovaryans matrisi, Ölçek faktörü, Güvenirlik

\section{Investigation of reliability of the variance covariance matrices obtained from GIPSY-OASIS II v6.4 software for precise point positioning}

\begin{abstract}
In recent years Precise Point Positioning (PPP) technique is one of the most important subjects in Geomatic Engineering. PPP technique needs only one Global Navigation Satellite Systems (GNSS) receiver thus users have preferred it instead of traditional relative positioning technique for several applications. Nowadays, scientific software generally has been used for PPP solutions and the Variance covariance (VCV) matrices estimated from software are very optimistic. The formal errors estimated from VCV matrices have major effects on statistical interpretation. VCV matrices derived from GNSS processing software play important role for deformation analysis and scientists sometimes need to scale VCV matrices. In this study, 10 continuously operating International GNSS Service (IGS) reference stations have been considered for 11 days dated 2014. All points have been analyzed by GIPSY-OASIS II v6.4 scientific software. It is aimed to estimate scale factor for the PPP results obtained from GIPSY-OASIS II v6.4 with considering different session durations as 2, 4, 6, 8, 12 and 24 hours. According to the results, the values of the scale factors raise depending on the raises in respect of session duration.
\end{abstract}

Keywords: Precise point positioning, GIPSY-OASIS II v6.4, Variance covariance matrix, Scale factor, Reliability 


\section{Giriş}

Küresel Navigasyon Uydu Sistemleri (Global Navigation Satellite Systems - (GNSS)) yapay uydulardan uzay geriden kestirme tekniğine dayalı olarak geliştirilmiş bir konum belirleme yöntemidir. Jeodezik çalışmalarda her türlü hava koşulunda çalışabilmesi, noktaların birbirini görmesini gerektirmemesi, gece-gündüz gözlem yapılabilmesi ve hassas konum bilgisinin elde edilebilmesi gibi katkılarından dolayı günümüzde farklı nitelikteki pek çok uygulamada sıklıkla tercih edilmektedir. GNSS ölçme yöntemi, temel jeodezik ağların oluşturulması, kadastral çalışmalar, batimetrik ölçmeler, tektonik hareketlerin modellenmesi, deformasyonların izlenmesi, çeşitli mühendislik uygulamaları vb. gibi cm, mm duyarlılığında hassas konum doğruluğu gerektiren çalışmalarda etkin biçimde kullanılmaktadır (Gao, Wojciechowski \& Chen, 2005; Calais, Han, DeMets \& Nocquet, 2006; Dixon, 2006; Ohta, Meilano, Sagiya, Kimata \& Hirahara, 2006; Ohta vd., 2008; Larson \& Miyazaki, 2008; Geng, Teferle, Meng \& Dodson, 2010; Reilinger, McClusky, Paradissis, Ergintav \& Vernant, 2010; Doğan, Tunalığlu, Erdoğan \& Öcalan, 2018). Bu çalışmalarda genellikle konumu iyi bilinen referans noktalarına dayalı olarak yeni noktaların koordinatlarının belirlenmesinde bağıl konum belirleme yöntemi kullanılmasına karşın, günümüzde bu yönteme alternatif hassas mutlak konum belirleme (Precise Point Positioning (PPP)) tekniği de sıklıkla tercih edilir olmuştur. PPP tekniği tek bir GNSS alıcısı ile başta uydu yörünge-saat düzeltme bilgileri ile diğer hata kaynaklarına ilişkin düzeltme modellerinin kullanıldığı mutlak konumlama tekniğidir. Bu teknikte bağıl konum belirleme tekniklerinden farklı olarak koordinatı bilinen bir ya da daha fazla referans istasyonuna gereksinim duyulmamaktadır. Zumberge, Heflin, Jefferson, Watkins ve Webb (1997) bu teknikle başka bir alıcıya ihtiyaç duymaksızın, kod ve faz ölçülerinin lineer kombinasyonlarının kullanılmasıyla cm hassasiyetinde konum doğruluğu elde edilebileceğini ortaya koymuştur. Kouba ve Hèroux (2001) ve Gao ve Shen (2001) GNSS hata kaynaklarının elimine edilmesinde uydu ve alıcı arasındaki fark alma tekniklerini kullanmak yerine, dış düzeltme unsurları olarak adlandırılan ürün ve modeller kullanmıştır. Günümüzde PPP tekniği ile gerçekleştirilen 24 saatlik çözümlerde yatay koordinat bileşenlerinde 2-3 mm, düşey koordinat bileşeninde ise $5 \mathrm{~mm}$ hassasiyetinde konum bilgisine ulaşılabilmektedir (Wang, 2013). Her ne kadar tamsayı belirsizliği çözümü aşamasında uzun yakınsama süresi ihtiyacı olsa da bu yöntem ile elde edilen doğruluğun, bağıl konum belirlemeye alternatif olabileceği vurgulanmaktadır (Tekiç, 2009; Öcalan, 2015).

GNSS yöntemiyle elde edilen konum bilgilerinin doğruluğu çözüm tekniğiyle birlikte ölçüm yöntemi, çevresel ve atmosferik etkiler, manyetik fırtınalar ve kullanılan yazılım gibi etkenlere bağlıdır. İstenilen doğruluğa ulaşılabilmesi için hata kaynaklarının çözüm aşamasında veya öncesinde giderilmesi gerekmektedir. Bundan dolayı verilerin değerlendirildiği yazılımın seçimi de önem arz etmektedir. Ancak her ne kadar yazılımlardan elde edilen konum doğrulukları istenilen seviyede olsa da Varyans-Kovaryans (VKV) matrisleri, fiziksel korelasyonların ihmal edilmesi, sistematik hataların modellenememesi ve epoklar arası korelasyonların dikkate alınmamasıyla çözüm aşamasında sadece blok-diyagonal matrislerin hesaba katılmasından dolayı iyimser sonuçlar vermektedir (Ananga, Coleman \& Rizos, 1994; McClusky vd., 2000; Nocquet, Calais \& Nicolon, 2002; Geirsson, 2003; Kashani, Wielgosz \& Grejner-Brzezinska, 2004). Bu durum VKV matrislerine dayalı istatistiksel testlerin yanlış yorumlanmasına sebep olmaktadır. Yorumlamalarda doğru sonuçların elde edilebilmesi için VKV matrislerinin daha gerçekçi elde edilmesi gerekmektedir. Varyans bileşen kestirimi, standartlaştırma ve ölçekleme gibi farklı yöntemler kullanılarak daha gerçekçi VKV matrisi elde edilmesiyle ilgili birçok çalışma gerçekleştirilmiştir (Ananga vd., 1994; Han \& Rizos, 1995; Geirsson, 2003; Kashani vd., 2004; Li, Shen \& Lou, 2011; Li, Lou \& Shen, 2015; Çetin, Aydın \& Doğan, 2018; Erdoğan \& Doğan, 2019). Geirsson (2003), Bernese yazılımından hesaplanan her bir koordinat bileşeni için ayrı ayrı ölçek faktörlerini (ÖF) hesaplamıştır. Çalışmada ÖF değerleri doğu bileşeni için 4.2, kuzey bileşeni için 3.9 ve yukarı bileşen için 2.3 olarak belirlense de yatay bileşenler için 4, düşey bileşen için 2.5 değerleri seçilmiştir. Kashani vd. (2004) çalışmasında Geirsson (2003)'ün aksine her bileşen için ayrı bir kestirim yapmak yerine tüm bileşenler için tek bir değer belirlemiştir. Bernese ve Gamit yazılımları için ÖF değerleri sırasıyla 23 ve 1.9 olarak belirlenmiştir. Han ve Rizos 
(1995)'de ise epok korelasyonları dikkate alınarak standartlaştırma işlemi gerçekleştirilmiştir. Standartlaştırılan VKV matrislerinin $\% 4$ oranında iyileştiğinin vurgulanmasına rağmen yine de bu matrislerin ölçeklenmesi gerektiği vurgulanmıştır. Çetin vd. (2018) de bir ölçekleme çalışması gerçekleştirmiştir. Bernese ve Gamit yazılımları ile elde edilen çözüm sonuçlarının dikkate alındığı bu çalışmada ÖF değerleri sırasıyla 3.82 ve 0.38 olarak hesaplanmıştır. Erdoğan ve Doğan (2019) Bernese v5.2 yazılımı sonuçlarının ölçeklendirilmesi amacıyla gözlem süresine bağlı olarak logaritmik bir fonksiyon ortaya koymuş̧ur.

Literatürdeki birçok çalışmada bağıl konum belirleme yöntemiyle elde edilen VKV matrislerinin güvenirliği araştırılmıştır. Bu çalışmada ise PPP çözümlerinden elde edilen VKV matrislerinin güvenirliğinin araştırılması amaçlanmıştır. Bunun için GIPSY-OASIS II v6.4 yazılımı kullanılarak 10 adet sürekli gözlem yapan GNSS istasyonuna ait 11 günlük veriler bu araştırmada kullanılmıştır. Çalışmada yer merkezli kartezyen dik koordinat sisteminde gerçekçi VKV matrislerinin elde edilebilmesi için ÖF değerleri kestirilmiştir. Ayrıca, koordinatlara ait zaman serilerindeki uyuşumsuz ölçüleri belirlemek için Medyan ve ÖF kestirimi aşamasında ise modelden uzaklaşan ölçüleri dişlamak için Pope test yöntemi kullanılmıştır. Çalışmanın ikinci bölümünde, PPP yönteminin matematiksel modeli ve hata kaynakları açıklanmaktadır. Üçüncü bölümde, ÖF değerlerinin kestirimi için kullanılan veri analiz modelleri ortaya konmuş, dördüncü bölümde, ÖF analizleri incelenmiştir. Beşinci bölümde ise çalışmanın sonuçlarına yer verilmektedir.

\section{PPP Matematiksel Modeli ve Hata Kaynakları}

PPP tekniği tek bir GNSS alıcısı kullanılarak mutlak konumlama ile cm seviyesinde konum doğruluğunun elde edilmesini sağlayan bir yöntemdir. PPP yöntemi ilk olarak Zumberge vd. (1997)'de ortaya konulmuştur. Kouba ve Hèroux (2001) PPP yönteminde kod ve faz ölçülerinin lineer kombinasyonlarını ve Uluslararası GNSS Servisinin (International GNSS Service IGS) sağladığı hassas yörünge ve saat ürünlerini kullanarak PPP’nin temel matematiksel modelini ortaya koymuşlardır. Bu modelde hem taşıyıcı faz hem de kod gözlemlerinin birlikte kullanılması, yüksek doğrulukla nokta konumlama için çok önemlidir. PPP tekniğinde geleneksel anlamda iyonosfer kaynaklı hataların etkisini azaltmak için çift frekanslı GPS gözlem modelleri benimsenmiştir. Buna göre iyonosfer-bağımsız kod ve taşıyıcı faz gözlemleri, L3 olarak bilinen kombine edilmiş iyonosfer-bağımsız yapıya dönüştürülmektedir. Böylece GPS sinyalinin iyonosfer tabakasında uğradığı kayıklıklar (refraksiyon bias), büyük oranda elimine edilmektedir (Öcalan, 2015). Eşitlik (1) ve (2)’de Kouba ve Hèroux (2001) tarafından verilen PPP yönteminin basitleştirilmiş matematiksel modeli görülmektedir.

$\ell_{p}=\rho+c(d T-d t)+T_{r}+\varepsilon_{p}$

$\ell_{\Phi}=\rho+c(d T-d t)+T r+N \lambda+\varepsilon_{\Phi}$

Burada; $\ell_{p}$ ve $\ell_{\Phi}$ sırasıyla iyonosfer bağımsız kod ve faz ölçülerini, $\rho$; uydu ve alıcı arasındaki geometrik mesafeyi, $d T$ ve $d t$ sırasıyla alıcı ve uydu saat hatalarını, $T r$, troposferik gecikmeyi, $N$, faz ölçülerinin başlangıç tamsayı belirsizliğini, $\varepsilon_{p}$ ve $\varepsilon_{\Phi}$ kod ve faz ölçülerindeki gürültüleri ifade etmektedir.

Uydu ve alıcı arasında hesaplanan geometrik mesafe $\rho$, bir t epoğunda yayınlanan uydu konumu $\left(\mathrm{X}_{\mathrm{S}}, \mathrm{Y}_{\mathrm{S}}, \mathrm{Z}_{\mathrm{S}}\right)$ ile alıcı $\left(\mathrm{X}_{\mathrm{r}}, \mathrm{Y}_{\mathrm{r}}\right.$, $\mathrm{Z}_{\mathrm{r}}$ ) konumunun fonksiyonu olarak (3) eşitliğiyle hesaplanmaktadır.

$\rho=\sqrt{\left(X_{S}-X_{r}\right)^{2}+\left(X_{S}-Y_{r}\right)^{2}+\left(Z_{S}-Z_{r}\right)^{2}}$

PPP çözümlerinin doğruluğunun arttırılabilmesi için hata kaynaklarının ürünler yardımıyla veya modellenerek giderilmesi 
gerekmektedir. Uydu saat hatası ve anten faz merkezi kayıklığı gibi hatalar farklı kurum ve kuruluşlar tarafından üretilen ürünler yardımıyla giderilebilirken kutup gezinmesi ve karasal gelgit gibi hataların modellenmesi gerekmektedir. Rizos, Janssen, Roberts ve Grinter (2012), PPP yöntemindeki hata kaynaklarını Tablo 1'deki şekilde özetlemiştir.

Tablo 1: PPP hata kaynakları ve düzeltme modelleri (Rizos vd., 2012)

\begin{tabular}{ll}
\hline Hata Kaynakları ve Düzeltme Modelleri & Düzeltme Türü \\
\hline & Hassas uydu saat düzeltmeleri \\
& Uydu anteni faz merkezi offset değerleri \\
& Uydu anteni faz merkezi kayılıkları \\
& Hassas uydu yörüngeleri \\
& Diferansiyel grup gecikmeleri \\
& Görelilik koşulu \\
Uydu Kaynaklı Hatalar & Uydu anteni faz dönmesi \\
& Alıcı anteni faz merkezi offset değerleri \\
& Alıcı anteni faz merkezi kayıklıkları \\
& Alıcı anteni faz dönmesi \\
\hline \multirow{2}{*}{ Alıcı Kaynaklı Hatalar } & Katı yeryuvarı gelgiti \\
& Okyanus yüklemesi \\
& Kutup gelgiti \\
\multirow{3}{*}{ Jeofiziksel Modeller } & Plaka tektonik hareketleri \\
& Troposferik gecikme \\
& İyonosferik gecikme \\
\hline \multirow{2}{*}{ Atmosferik Modelleme } &
\end{tabular}

\section{Veri Analizi}

Bu çalışma, 10 adet sürekli gözlem yapan sabit GNSS referans istasyonunun, 2014 yılının 28. ve 38. günleri arasına denk gelen 11 günlük verilerini içermektedir (Şekil 1). Bu dönemin seçilme sebebi "Kp-indeks" değerlerinin uygun olmasıdır (Şekil 2).

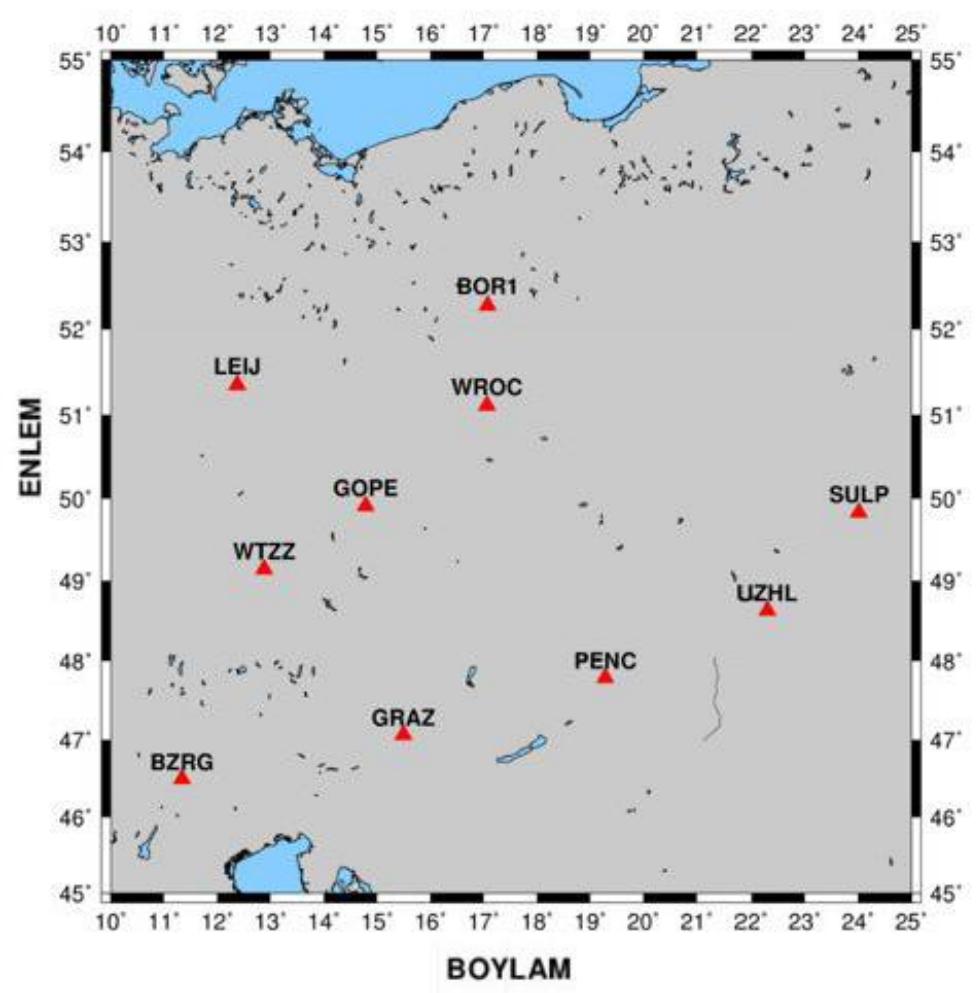


Kp-indeks değeri dünya üzerine dağılmış manyetometrelerle 3 saatlik gözlemler sonucunda elde edilmektedir. 0 ile 9 arasında yer alan bu değerler küresel ölçekte jeomanyetik etkiyi ifade etmektedir. 0, çok az etkiyi gösterirken; 9 , yüksek derecede jeomanyetik firtınaların gerçekleştiğini göstermektedir. 4 değeri sınır değer olarak ifade edilmektedir (URL-1). Bu değerler Ocak ayının sonu ile Şubat ayının başlangıç kısımlarında düşük olarak gözlemlenmiştir. Noktaların günlük verileri Scripps Orbit and Permanent Array Center (SOPAC) arşivinden elde edilerek Jet Propulsion Laboratory (JPL) tarafından geliştirilen GIPSY-OASIS II v6.4 yazılımı kullanılarak değerlendirilmiştir. Bu çalışmada sadece GPS verileri değerlendirilmiştir ve değerlendirilen istasyonlar seçilirken enlemin etkisi göz önünde bulundurularak orta kuşakta bulunan istasyonlar tercih edilmiştir. GPS uydularının yörünge tasarımından dolayı ekvatora yakın olan noktalarda, uydular Kuzey-Güney doğrultusunda hareket etmektedir. Bu durum orta kuşakta bulunan noktalarda görülmemektedir (Blewitt, 1997). Noktaların aynı özellikte olmaları bakımından yaklaşık olarak yakın enlemdeki noktalar seçilmiştir. Noktaların yaklaşık koordinat bilgileri Tablo 2'de verilmektedir.

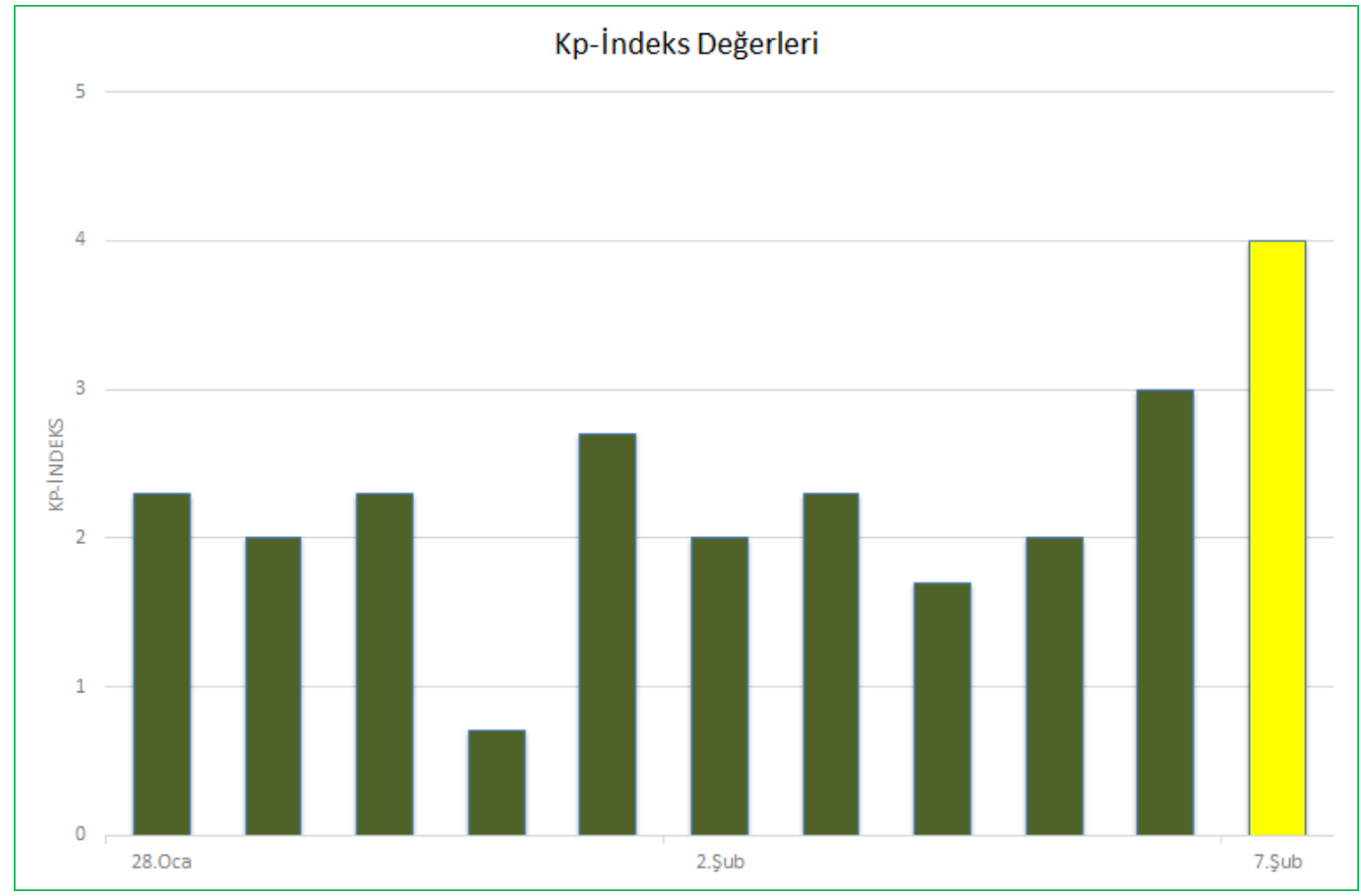

Şekil 2: Çalışma dönemine ait Kp-Indeks değerleri (URL-1)

Tablo 2: Noktaların yaklaşık enlem, boylam ve elipsoidal yükseklik değerleri (WGS84)

\begin{tabular}{c|ccc}
\hline \multirow{2}{*}{ Nokta Adı } & \multicolumn{3}{c}{ Coğrafi Koordinat Değerleri } \\
\cline { 2 - 4 } & Enlem (derece) & Boylam (derece) & Elipsoidal Yükseklik (m) \\
\hline BOR1 & 52.1002 & 17.0668 & 124 \\
\hline BZRG & 46.4990 & 11.3368 & 329 \\
\hline GOPE & 49.9137 & 14.7856 & 593 \\
\hline GRAZ & 47.0420 & 15.4935 & 538 \\
\hline LEIJ & 51.2114 & 12.2222 & 292 \\
\hline PENC & 47.7896 & 19.2815 & 371 \\
\hline SULP & 49.8356 & 24.0145 & 232 \\
\hline UZHL & 48.6320 & 22.2976 & 181 \\
\hline WROC & 51.1131 & 17.0619 & 666 \\
\hline WTZZ & 49.1442 & 12.8789 & \\
\hline
\end{tabular}

Literatürde gerçekleştirilen çalışmalar incelendiğinde GNSS uygulamalarında hesaplanan koordinat değerlerine ilişkin doğruluk analizi çalışmalarında tekrarlılıklardan hesaplanan Karesel Ortalama Hata (Root Mean Square-RMS) değerleri kullanılmıştır ve GPS çözümlerinin doğruluğunun gözlem süresine bağlı olduğu görülmektedir (Eckl, Snay, Soler, Cline \& 
Mader, 2001; Soler, Michalak, Weston, Snay \& Foote, 2006; Şanlı \& Engin, 2009). Bu durumda GIPSY-OASIS II v6.4 yazılımıyla elde edilen standart sapma değerlerinin de gözlem süresine bağlı olarak değişmesi beklenir. Dolayısıyla, bu çalışmada VKV matrislerinin güvenirliği araştırıldığından ÖF değerlerinin de gözlem süresine bağlı olup olmadığını belirleyebilmek için GPS verileri 2, 4, 6, 8, 12 ve 24 saatlik alt gözlem sürelerine bölünmüştür.

GIPSY-OASIS II v6.4, PPP çözüm yaklaşımını kullanan bilimsel bir yazılımdır. PPP tekniğinde hassas nokta konum bilgisine ulaşabilmek için Bölüm 2'de verilen hata kaynaklarının giderilmesi ve modellenmesi gerekmektedir. Bu araştırmada GPS verilerinin değerlendirilmesi ve analizi aşamasında; JPL tarafından üretilen hassas uydu yörünge ve saat bilgileri kullanılmıştır. Alıcı saat hatası modellenmiştir. Alıcı ve uyduların anten faz merkezi kayıklıkları giderilmiştir. Karasal ve okyanus gelgiti etkileri göz önünde bulundurulmuştur. Troposferik 1slak gecikme, doğu ve kuzey bileşenlerindeki gradyentler kestirilmiştir. Ayrıca ikinci mertebeden iyonosferik etki de göz önünde bulundurularak, "iri” modeli yardımıyla giderilmiştir (Kedar, Hajj, Wilson \& Heflin, 2003). GIPSY-OASIS II v6.4 yazılımı çözüm stratejileri için ayrıntılı bilgiye URL-2’den ulaşılabilmektedir.

Çözümler ITRF2008 datumunda gerçekleştirilmiştir. Yazılım sonuç ürün olarak yer merkezli kartezyen sisteminde dik koordinatları, standart sapmaları ve korelasyon katsayılarını vermektedir. Bu çalışmada daha gerçekçi VKV matrislerinin elde edilmesi amaçlanmaktadır. GPS’in doğruluğunun araştırıldığı çalışmalarda doğruluk, koordinat tekrarlılıklarından hesaplanan RMS değerleri ile ilişkilendirilmiştir (Eckl vd., 2001; Soler vd., 2006; Şanlı \& Engin 2009; Tut, Şanlı, Erdoğan \& Hekimoğlu, 2013; Doğan, Uludağ \& Demir, 2014). Koordinat serilerinden elde edilen RMS değerlerinin doğruluğu yansıttığı düşünüldüğü için elde edilen standart sapmaların da RMS değerlerine yakın olması beklenir. Bundan dolayı PPP çözümleri için uygun ÖF değerini belirleyebilmek için (4) ve (5) eşitliklerindeki modeller oluşturulmuştur:

$R M S_{i, j, k}=\ddot{O} F \times S T D_{i, j, k, t}$

$\ddot{O} F=\frac{R M S_{i, j, k}}{S T D_{i, j, k, t}}$

Burada RMS; koordinat serilerinden elde edilen RMS değerlerini; STD, yazılımdan elde edilen standart sapmaları ve ÖF ölçek faktörü değerini ifade etmektedir. (i: istasyon, j: koordinat bileşeni, k: gözlem süresi, t: gözlem sayıs1)

RMS değerleri (6) eşitliği kullanılarak elde edilmiştir. Bu aşamada koordinat serilerindeki uyuşumsuz olan ölçüler Medyan yöntemi kullanılarak elimine edilmiştir (Eşitlik 7). Medyan yöntemi \%50 kırılma noktasıyla en güvenilir robust yöntemlerden bir tanesidir (Hampel, Ronchetti, Rousseeuw \& Stahel, 2011; Hekimoğlu, 2005).

$R M S=\sqrt{\frac{\sum_{i=1}^{n}\left(X_{i}-X_{R}\right)^{2}}{n}}$

Burada RMS, koordinat serilerinden elde edilen RMS değerlerini; $X_{R}$, referans koordinat değerini; $X_{i}$, i. koordinat değerini ve $n$, ölçü sayısını ifade etmektedir. RMS değerleri her bir nokta, her bir bileşen ve her bir gözlem süresi için ayrı ayrı hesaplanmıştır. Referans değer olarak 24 saatlik koordinat değerlerinin ortalaması kullanılmıştır.

med $=$ median $(\mathbf{X})$

$\operatorname{mad}=\left\{\begin{array}{c}1.2533 \times \frac{1}{n} \times \sum \mid \mathbf{X}-\text { med } \mid, \text { median } \mid \boldsymbol{X}-\text { med } \mid=0 \\ 1.4826 \times \text { median } \mid \mathbf{X}-\text { med } \mid, \text { median } \mid \boldsymbol{X}-\text { med } \mid \neq 0\end{array}\right.$ 
Burada X, düzeltmeleri; med, düzeltmelerin ortanca değerini ve n, ölçü sayısını ifade etmektedir. Bu yöntemde düzeltmeler $3 \times$ mad değeri ile karşılaştırılır. Sınır değerden küçük veya eşit olan ölçüler uyuşumlu, büyük olan ölçüler uyuşumsuz olarak kabul edilir. Şekil 3-5 medyan yöntemine göre uyuşumsuz ölçü analizi sonucunda hesaplanan RMS değerlerini göstermektedir.

(4) ve (5) eşitliklerinde ÖF değerleri kestiriminde En Küçük Kareler (EKK) yöntemi kullanılmıştır (Eşitlik 8). Ayrıca, ilgili modelde farklılık gösteren ölçüler Pope test yöntemiyle çözümden çıkarılmıştır (Eşitlik 9). Pope test yönteminde her bir ölçüye ait düzeltme değerleri için test değerleri hesaplanır. Tau dağılımlı sınır değerden büyük olan en büyük test değerine sahip ölçü dengelemeden çıkarılır. İteratif olarak uyuşumsuz ölçü kalmayıncaya kadar bu işlem tekrarlanır (Pope, 1976).

$x=\left(\mathrm{A}^{\mathrm{T}} \mathrm{A}\right)^{-1} \mathrm{~A}^{\mathrm{T}} \mathbf{l}$

$\mathbf{A}=\left[\begin{array}{c}S T D_{1, j, k, t} \\ \vdots \\ S T D_{n, j, k, t}\end{array}\right]$ (4) nolu eşitlik için A katsayıllar matrisi

$\mathbf{A}=\left[\begin{array}{c}1 \\ \vdots \\ 1\end{array}\right]$ (5) nolu eşitlik için $\mathbf{A}$ katsayılar matrisi

$\overline{\mathbf{v}}=\mathbf{A x}-\mathbf{l}$

$\mathbf{I}=\left[\begin{array}{c}R M S_{1, j, k} \\ \vdots \\ R M S_{n, j, k}\end{array}\right]$ (4) nolu eşitlik için I ölçü vektörü

$\mathbf{I}=\left[\begin{array}{c}\frac{R M S_{1, j, k}}{S T D_{1, j, k, t}} \\ \vdots \\ \frac{R M S_{n, j, k}}{S T D_{n, j, k, t}}\end{array}\right]$ (5) nolu eşitlik için I ölçü vektörü

$\mathbf{Q}_{\overline{\mathbf{v}} \overline{\mathrm{v}}}=\mathbf{E}-\mathbf{A}\left(\mathbf{A}^{\mathrm{T}} \mathbf{A}\right)^{-1} \mathbf{A}^{\mathrm{T}}$

$S_{0}=\sqrt{\frac{\overline{\mathbf{v}} \overline{\mathbf{v}}}{n-1}}$

$\tau_{i}=\frac{\left|\bar{v}_{i}\right|}{S_{0} \times \sqrt{q_{\bar{v}_{i} \bar{v}_{i}}}}$

Burada A, katsayılar matrisini; l, ölçü vektörünü; $\overline{\mathbf{v}}$, düzeltme vektörünü; $\mathbf{E}$, birim matrisi; n, ölçü sayısını; $S_{0}$, birim ağırlıklı sonsal standart sapmay1; $\mathbf{Q}_{\overline{\mathbf{v}} \overline{\mathrm{v}}}$, düzeltmelerin kofaktör matrisini; $q_{\bar{v}_{i} \bar{v}_{i}}$, i. düzeltmenin kofaktör elemanını; $\tau_{i}$, i. düzeltmenin test değerini ve $\mathrm{x}$, bilinmeyen olan ÖF değerini ifade etmektedir. 


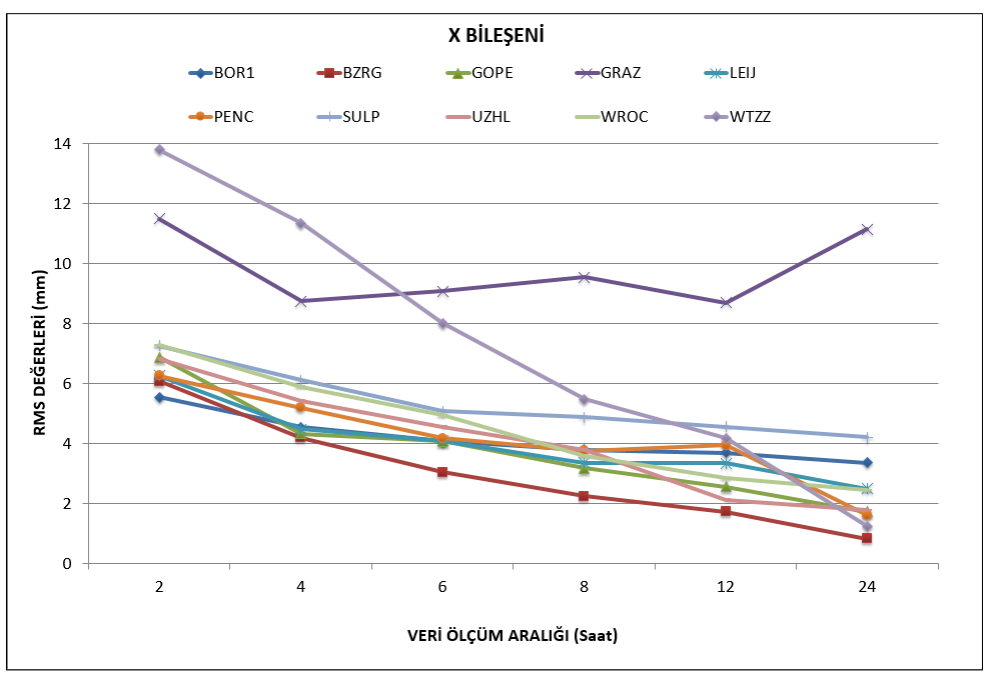

Şekil 3: Noktaların X koordinat bileşenleri için RMS değerleri

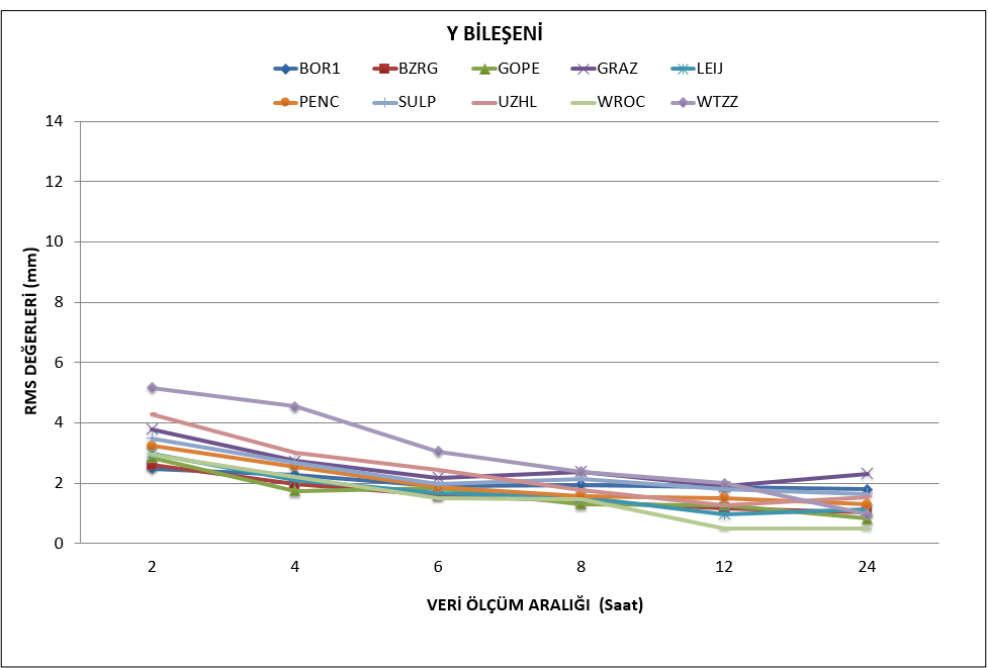

Şekil 4: Noktaların Y koordinat bileşenleri için RMS değerleri

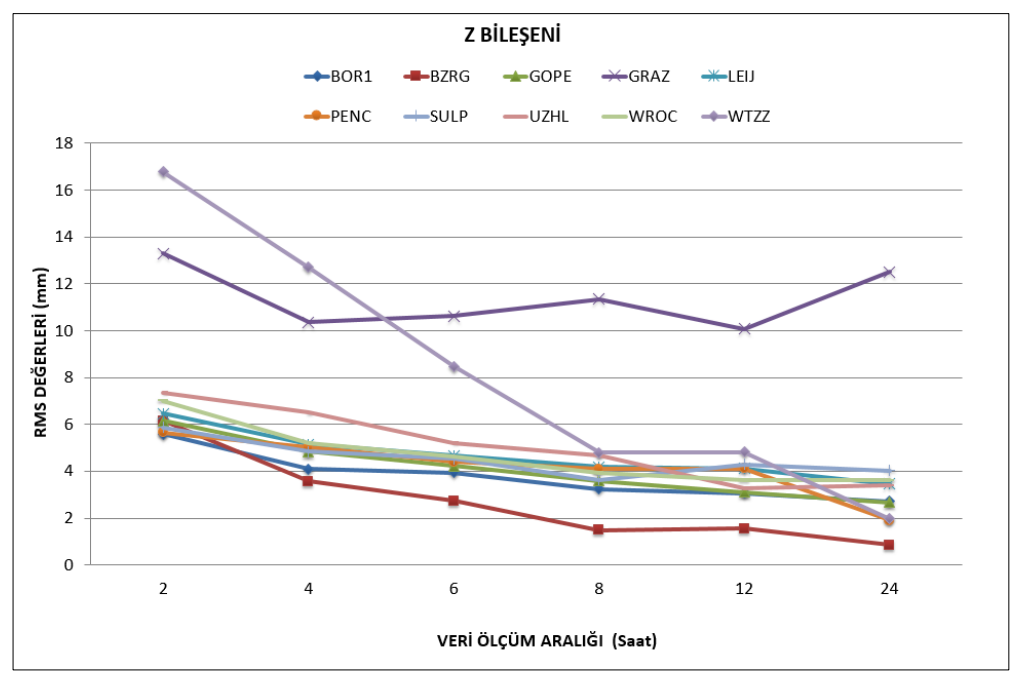

Şekil 5: Noktaların Z koordinat bileşenleri için RMS değerleri 


\section{4. Ölçek Faktörü Analizleri}

$\mathrm{Bu}$ çalışmada koordinat zaman serilerinden hesaplanan RMS değerleri ile yazılımdan kestirilen standart sapmalar arasında ilişki kuracak iki farklı model karşılaştırılmıştır. Ayrıca bu iki farklı modelde ÖF değerinin hesabı için iki farklı strateji kullanılmıştır. Birinci stratejide ÖF değerleri, her bir istasyonun her bir gözlem süresi (veri seti) için ayrı ayrı hesaplanırken, ikinci stratejide esas olarak gözlem süreleri dikkate alınarak tüm istasyonların verilerine dayalı hesaplama gerçekleştirilmiştir. Bunun yanında hangi modelin daha uygun sonuçlar verdiğini belirleyebilmek için norm değerleri hesaplanmıştır. Norm değeri, farklı yöntem ve stratejilerin performanslarını karşılaştırmak için kullanılan matematiksel bir büyüklüktür. Farklı yöntem ve stratejilerden elde edilen vektör veya matrisler, beklenen vektör veya matrislerle karşılaştırılmaktadır.

Norm değeri; bu uygulamada, farklı model ve stratejilerden hesaplanan ÖF değerleri kullanılarak ölçeklendirilen standart sapma değerleri ile koordinat serilerinden hesaplanan RMS değerleri arasındaki farklar kullanılarak belirlenmiştir (Eşitlik $10)$.

$N=\sqrt{\sum_{t=1}^{n}\left(\ddot{O} S T D_{i, j, k, t}-R M S_{i, j, k}\right)^{2}}$

Burada RMS, GPS koordinat zaman serilerinden hesaplanan RMS değerlerini; ÖSTD, ölçeklendirilen standart sapma değerlerini ve N, norm değerini ifade etmektedir (i: istasyon, j: koordinat bileşeni, k: gözlem süresi, t: gözlem sayısı).

Tablo 3: Birinci modelde her bir veri seti için sonuçlar

\begin{tabular}{c|cc|cc|cc|cc|cc|cc|c} 
& \multicolumn{2}{|c|}{$\mathbf{2}$ saat } & \multicolumn{2}{c|}{ 4 saat } & \multicolumn{2}{c|}{ 6 saat } & \multicolumn{2}{c|}{$\mathbf{8}$ saat } & \multicolumn{2}{|c|}{ 12 saat } & \multicolumn{2}{|c|}{ 24 saat } \\
\hline Nokta & ÖF & $\begin{array}{c}\text { Norm } \\
(\mathbf{m m})\end{array}$ & ÖF & $\begin{array}{c}\text { Norm } \\
(\mathbf{m m})\end{array}$ & ÖF & $\begin{array}{c}\text { Norm } \\
(\mathbf{m m})\end{array}$ & ÖF & $\begin{array}{c}\text { Norm } \\
(\mathbf{m m})\end{array}$ & ÖF & $\begin{array}{c}\text { Norm } \\
(\mathbf{m m})\end{array}$ & ÖF & $\begin{array}{c}\text { Norm } \\
(\mathbf{m m})\end{array}$ \\
\hline BOR1 & 0.80 & 14.94 & 0.93 & 9.21 & 1.07 & 5.40 & 1.09 & 6.12 & 1.30 & 4.95 & 1.67 & 3.53 \\
\hline BZRG & 0.77 & 19.19 & 0.77 & 5.74 & 0.73 & 3.22 & 0.56 & 4.21 & 0.61 & 2.09 & 0.48 & 2.04 \\
\hline GOPE & 0.79 & 20.84 & 0.84 & 4.82 & 0.96 & 3.30 & 0.90 & 1.71 & 0.95 & 1.49 & 0.39 & 7.53 \\
\hline GRAZ & 1.81 & 29.59 & 2.05 & 15.62 & 2.60 & 14.15 & 3.20 & 12.89 & 3.55 & 9.72 & 6.31 & 8.97 \\
\hline LEIJ & 0.90 & 15.10 & 1.02 & 4.84 & 1.14 & 2.52 & 1.16 & 1.47 & 1.34 & 3.22 & 1.60 & 1.13 \\
\hline PENC & 0.76 & 16.16 & 1.00 & 5.31 & 1.01 & 2.98 & 1.09 & 2.41 & 1.36 & 2.38 & 0.92 & 1.22 \\
\hline SULP & 0.85 & 24.39 & 1.07 & 12.37 & 1.17 & 6.85 & 1.22 & 7.21 & 1.57 & 4.00 & 2.08 & 2.13 \\
\hline UZHL & 0.95 & 45.15 & 1.21 & 5.44 & 1.23 & 4.33 & 1.21 & 3.08 & 0.98 & 2.25 & 1.38 & 2.54 \\
\hline WROC & 1.04 & 19.74 & 1.18 & 10.19 & 1.25 & 6.69 & 1.16 & 2.25 & 1.19 & 3.76 & 1.50 & 2.53 \\
\hline WTZZ & 2.12 & 33.63 & 2.50 & 12.66 & 2.14 & 5.11 & 1.56 & 5.13 & 1.70 & 1.09 & 0.90 & 1.48 \\
\hline Toplam & & 238.74 & & 86.19 & & 54.55 & & 46.49 & & 34.95 & & 33.10 \\
\hline
\end{tabular}

Tablo 3 ve 4'te birinci model için hesaplanan ÖF ve norm değerleri görülmektedir. Norm değerlerinin küçük olması koordinat zaman serilerinden elde edilen RMS değerlerinin, ölçeklendirilen değerlere yakın olduğunu göstermektedir. Bu şekilde farklı stratejilerden elde edilen norm değerleri karşılaştırılarak hangi stratejinin daha uygun sonuç verdiğine karar verilebilir. Tablo 3'e göre GRAZ ve WTZZ noktalarında ÖF değerlerinin diğer noktalarda hesaplanan değerlere göre daha büyük olduğu görülmektedir. Ayrıca diğer noktalarda gözlem süresi arttıkça ÖF değerlerinde de bir artış olduğu görülmektedir. Nokta bazlı ÖF değerinin hesaplanmasında genel anlamda gözlem süresine bağlı bir artış görülse de noktalar arasında farklılıklar bulunmaktadır. Bu bağlamda tüm noktaları içeren gözlem süresine bağlı ÖF değerinin kestirilmesi daha anlamlı olacaktır.

Tablo 4'te birinci model için gözlem sürelerine göre hesaplanan ÖF ve norm değerleri verilmektedir. Akademik yazılımlarda gözlem süresi arttıkça analiz sonucunda elde edilen standart sapmalar küçülmektedir. Bu nedenle gözlem süresi arttıkça ÖF değerlerinin de arttığg görülmektedir. 
Tablo 4: Birinci modelde her bir gözlem süresi için sonuçlar

\begin{tabular}{c|cccccc}
\multirow{2}{*}{ ÖF } & 2 Saat & 4 Saat & 6 Saat & 8 Saat & 12 Saat & 24 Saat \\
\cline { 2 - 7 } & 1.03 & 1.22 & 1.28 & 1.10 & 1.40 & 1.69 \\
\hline Norm $(\mathrm{mm})$ & 187.49 & 98.57 & 63.67 & 58.27 & 41.49 & 50.70 \\
\hline
\end{tabular}

Tablo 5, ikinci model için elde edilen ÖF ve norm değerlerini içermektedir. ÖF değerleri incelendiğinde bu modelde noktalara ait hesaplanan ÖF değerlerinde büyük farklılıklar olduğu görülmektedir. Bu noktalar birinci modelde de benzer etkiye sahip olan GRAZ ve WTZZ noktalarıdır. Bu durum noktalara özgü bozucu etkiler sebebiyle ortaya çıkabilmektedir. Tablo 6'da tüm gözlem süreleri dikkate alındığında gözlem süresi arttıkça ÖF değerlerinin de arttığı görülmektedir.

Çalışmada araştırılan iki modele ilişkin sonuçlar incelendiğinde birinci modeldeki norm değerlerinin yani beklenen değerden sapmaların daha küçük olduğu görülmektedir. Bu bağlamda analizlerde birinci model tercih edilebilir. Ayrıca, gözlem süresine göre gerçekleştirilen değerler tüm noktaların özelliklerini içerdiği için ÖF değerlerinin hesabında Tablo 4'de verilen değerler tercih edilebilir.

Tablo 5: Ikinci modelde her bir veri seti için sonuçlar

\begin{tabular}{|c|c|c|c|c|c|c|c|c|c|c|c|c|}
\hline & \multicolumn{2}{|c|}{2 saat } & \multicolumn{2}{|c|}{4 saat } & \multicolumn{2}{|c|}{6 saat } & \multicolumn{2}{|c|}{8 saat } & \multicolumn{2}{|c|}{12 saat } & \multicolumn{2}{|c|}{24 saat } \\
\hline Nokta & ÖF & $\begin{array}{c}\text { Norm } \\
(\mathbf{m m})\end{array}$ & ÖF & $\begin{array}{l}\text { Norm } \\
(\mathbf{m m})\end{array}$ & ÖF & $\begin{array}{l}\text { Norm } \\
(\mathbf{m m})\end{array}$ & ÖF & $\begin{array}{c}\text { Norm } \\
(\mathbf{m m})\end{array}$ & ÖF & $\begin{array}{c}\text { Norm } \\
(\mathbf{m m})\end{array}$ & ÖF & $\begin{array}{l}\text { Norm } \\
\text { (mm) }\end{array}$ \\
\hline BOR1 & 0.84 & 15.83 & 1.02 & 10.44 & 1.12 & 5.82 & 1.22 & 6.99 & 1.44 & 5.58 & 1.89 & 4.07 \\
\hline BZRG & 0.82 & 20.51 & 0.83 & 6.88 & 0.81 & 4.32 & 0.69 & 5.37 & 0.73 & 2.92 & 0.72 & 2.89 \\
\hline GOPE & 0.84 & 22.59 & 0.83 & 4.80 & 1.00 & 3.62 & 0.89 & 1.71 & 0.98 & 1.61 & 0.93 & 13.15 \\
\hline GRAZ & 1.71 & 31.04 & 1.88 & 17.30 & 2.28 & 17.45 & 2.82 & 15.98 & 3.05 & 12.53 & 5.40 & 11.73 \\
\hline LEIJ & 0.97 & 17.65 & 1.06 & 5.46 & 1.13 & 2.52 & 1.16 & 1.48 & 1.22 & 3.78 & 1.56 & 1.18 \\
\hline PENC & 0.81 & 17.37 & 1.00 & 5.36 & 0.98 & 3.16 & 1.04 & 2.77 & 1.28 & 2.82 & 1.01 & 1.45 \\
\hline SULP & 0.92 & 26.26 & 1.11 & 12.56 & 1.15 & 6.88 & 1.26 & 7.31 & 1.51 & 4.04 & 2.01 & 2.24 \\
\hline UZHL & 1.05 & 51.80 & 1.23 & 5.53 & 1.24 & 4.39 & 1.17 & 3.23 & 0.95 & 2.30 & 1.41 & 2.55 \\
\hline WROC & 1.07 & 20.38 & 1.19 & 10.22 & 1.19 & 7.04 & 1.14 & 2.29 & 0.98 & 5.05 & 1.22 & 3.39 \\
\hline WTZZ & 2.07 & 33.91 & 2.47 & 12.57 & 2.10 & 5.25 & 1.64 & 5.51 & 1.73 & 1.27 & 0.99 & 1.66 \\
\hline Toplam & & 257.32 & & 91.12 & & 60.44 & & 52.62 & & 41.90 & & 44.31 \\
\hline
\end{tabular}

Tablo 6: Ikinci modelde her bir gözlem süresi için sonuçlar

\begin{tabular}{c|cccccc}
\multirow{2}{*}{ ÖF } & 2 Saat & 4 Saat & 6 Saat & 8 Saat & 12 Saat & 24 Saat \\
\cline { 2 - 7 } & 1.11 & 1.26 & 1.29 & 1.16 & 1.39 & 1.74 \\
\hline Norm $(\mathrm{mm})$ & 191.0 & 98.9 & 63.7 & 57.3 & 41.5 & 51.2 \\
\hline
\end{tabular}

\section{Sonuçlar}

Bu çalışmada PPP çözümlerinden elde edilen VKV matrislerinin güvenirliği araştırılmıştır. Bu amaçla daha gerçekçi VKV matrisi elde edebilmek için iki farklı modelde iki farklı strateji kullanılarak ÖF değerleri hesaplanmıştır. Analiz sonuçları incelendiğinde birinci modelden hesaplanan değerlerin norm değerleri bazında ikinci modele göre daha iyi sonuç verdiği görülmüş̧ür. Ayrıca, PPP tekniğine ilişkin doğruluk fonksiyonlarının gözlem süresine bağlı olarak değiştiği düşünüldüğünde, gözlem süreleri dikkate alınarak tüm istasyonların verilerine dayalı gerçekleştirilen hesaplamalar dikkate alındığı durumlardaki ÖF değerlerinin kullanımının daha anlamlı olduğu görülmüştür. Sonuç olarak deformasyon analizi ve tektonik hareketlerin modellenmesi gibi istatistiksel analizlerde kullanılması için GIPSY-OASIS II v6.4 yazılımının VKV matrislerinin ölçeklendirilmesi gerekmektedir. Norm değerlerinin küçük olması durumu dikkate alındığında genel anlamda 
birinci modelden her bir gözlem süresine dayalı hesaplanan ÖF değerleri kullanılabilir.

\section{Teşekkür}

GIPSY-OASIS II v6.4 yazılımı ve hassas uydu yörünge ve saat bilgileri için NASA JPL'e, GPS verileri için SOPAC'a teşekkür ederiz. Ayrıca, Şekil 1 Generic Mapping Tools (GMT) yazılımı kullanılarak çizilmiştir (Wessel \& Smith, 1995).

\section{Kaynaklar}

Ananga, N., Coleman, R., \& Rizos, C. (1994). Variance-covariance estimation of GPS networks. Bulletin géodésique, 68(2), 77-87.

Blewitt, G. (1997). Basics of the GPS technique: observation equations. Geodetic applications of GPS, 10-54.

Calais, E., Han, J. Y., DeMets, C., \& Nocquet, J. M. (2006). Deformation of the North American plate interior from a decade of continuous GPS measurements. Journal of geophysical research: solid earth, 111(B6).

Çetin, S., Aydın, C., \& Doğan, U. (2018). Comparing GPS positioning errors derived from GAMIT/GLOBK and Bernese GNSS software packages: A case study in CORS-TR in Turkey. Survey Review, 1-11.

Dixon, K. (2006). StarFire: A global SBAS for sub-decimeter precise point positioning. In Proceedings of ION GNSS (pp. 26-29).

Doğan, U., Uludağ, M., \& Demir, D. O. (2014). Investigation of GPS positioning accuracy during the seasonal variation. Measurement, 53, 91-100.

Doğan, A. H., Tunalığlu, N., Erdoğan, B., \& Öcalan, T. (2018). Evaluation of the GPS Precise Point Positioning technique during the 21 July 2017 Kos-Bodrum (East Aegean Sea) Mw 6.6 earthquake. Arabian Journal of Geosciences, 11(24), 775.

Eckl, M. C., Snay, R. A., Soler, T., Cline, M. W., \& Mader, G. L. (2001). Accuracy of GPS-derived relative positions as a function of interstation distance and observing-session duration. Journal of geodesy, 75(12), 633-640.

Erdoğan, B., \& Doğan, A. H. (2019). Scaling of the variance covariance matrix obtained from Bernese software. Acta Geodaetica et Geophysica, 2(54), 197-211.

Gao, Y., \& Shen, X. (2001). Improving ambiguity convergence in carrier phase-based precise point positioning. In Proceedings of the 14th international technical meeting of the Satellite Division of the Institute of Navigation (ION GPS 2001), 1532-1539.

Gao, Y., Wojciechowski, A., \& Chen, K. (2005). Airborne kinematic positioning using precise point positioning methodology. Geomatica, 59(1), 29-36.

Geirsson, H. (2003). Continuous GPS measurements in Iceland 1999 - 2002 (Yüksek Lisans Tezi). University of Iceland, Akureyri, İzlanda.

Geng, J., Teferle, F. N., Meng, X., \& Dodson, A. H. (2010). Kinematic precise point positioning at remote marine platforms. GPS solutions, 14(4), 343-350.

Hampel, F. R., Ronchetti, E. M., Rousseeuw, P. J., \& Stahel, W. A. (2011). Robust statistics: the approach based on influence functions. John Wiley \& Sons.

Han, S., \& Rizos, C. (1995). Standardisation of the variance-covariance matrix for GPS rapid static positioning. Geomatics Research Australasia, 37-54.

Hekimoğlu, Ş. (2005). Do robust methods identify outliers more reliably than conventional test for outlier. Zeitschrift für Vermessungwesen, 3, 174-180.

Kashani, I., Wielgosz, P., \& Grejner-Brzezinska, D. A. (2004). On the reliability of the VCV Matrix: A case study based on GAMIT and Bernese GPS Software. GPS Solutions, 8(4), 193-199.

Kedar, S., Hajj, G. A., Wilson, B. D., \& Heflin, M. B. (2003). The effect of the second order GPS ionospheric correction on receiver positions. Geophysical Research Letters, 30(16).

Kouba, J., \& Héroux, P. (2001). Precise point positioning using IGS orbit and clock products. GPS solutions, 5(2), 12-28.

Larson, K. M., \& Miyazaki, S. I. (2008). Resolving static offsets from high-rate GPS data: the 2003 Tokachi-oki earthquake. Earth, planets and space, 60(8), 801-808.

Li, B., Shen, Y., \& Lou, L. (2011). Efficient estimation of variance and covariance components: a case study for GPS stochastic model 
evaluation. IEEE Transactions on Geoscience and Remote Sensing, 49(1), 203-210.

Li, B., Lou, L., \& Shen, Y. (2015). GNSS elevation-dependent stochastic modeling and its impacts on the statistic testing. Journal of Surveying Engineering, 142(2), 04015012.

McClusky, S., Balassanian, S., Barka, A., Demir, C., Ergintav, S., Georgiev, I., ... \& Kastens, K. (2000). Global Positioning System constraints on plate kinematics and dynamics in the eastern Mediterranean and Caucasus. Journal of Geophysical Research: Solid Earth, 105(B3), 5695-5719.

Nocquet, J., Calais, E., \& Nicolon, P. (2002). Reference frame activity: Combination of National (RGP) and Regional (REGAL) Permanent Networks Solutions with EUREF-EPN and the ITRF2000. In Proceedings of The EUREF 2002 Symposium, 398-404.

Ohta, Y., Meilano, I., Sagiya, T., Kimata, F., \& Hirahara, K. (2006). Large surface wave of the 2004 Sumatra-Andaman earthquake captured by the very long baseline kinematic analysis of 1-Hz GPS data. Earth, planets and space, 58(2), 153-157.

Ohta, Y., Ohzono, M., Miura, S., Iinuma, T., Tachibana, K., Takatsuka, K., Miyao, K., Sato, T., \& Umino, N. (2008). Coseismic fault model of the 2008 Iwate-Miyagi Nairiku earthquake deduced by a dense GPS network. Earth, planets and space, 60(12), 1197-1201.

Öcalan, T. (2015). GNSS Ă̆larında GPS Hassas Nokta Konumlama (GPS-PPP) Tekniği Yaklaşımlı Çözümler (Doktora Tezi). Yıldız Teknik Üniversitesi, Fen Bilimleri Enstitüsü, İstanbul, Türkiye.

Pope, A. J. (1976). The statistics of residuals and the detection of outliers (No. NOS-65-NGS-1).

Reilinger, R., McClusky, S., Paradissis, D., Ergintav, S., \& Vernant, P. (2010). Geodetic constraints on the tectonic evolution of the Aegean region and strain accumulation along the Hellenic subduction zone. Tectonophysics, 488(1-4), 22-30.

Rizos, C., Janssen, V., Roberts, C., \& Grinter, T. (2012). Precise point positioning: is the era of differential GNSS positioning drawing to an end?

Soler, T., Michalak, P., Weston, N. D., Snay, R. A., \& Foote, R. H. (2006). Accuracy of OPUS solutions for 1-to 4-h observing sessions. GPS solutions, 10(1), 45-55.

Şanl, D. U., \& Engin, C. (2009). Accuracy of GPS positioning over regional scales. Survey Review, 41(312), 192-200.

Tekiç, S. (2009). Accuracy Of GPS Precise Point Positioning (PPP) (Yüksek Lisans Tezi). Boğaziçi Universitesi, Deprem Araştırma Enstitüsü, İstanbul, Türkiye.

Tut, I., Şanlı, D. U., Erdoğan, B., \& Hekimoğlu, Ş. (2013). Efficiency of BERNESE single baseline rapid static positioning solutions with search strategy. Survey review, 45(331), 296-304.

Wang, G. Q. (2013). Millimeter-accuracy GPS landslide monitoring using Precise Point Positioning with Single Receiver Phase Ambiguity (PPP-SRPA) resolution: a case study in Puerto Rico. Journal of geodetic science, 3(1), 22-31.

Wessel, P., \& Smith, W. H. (1995). New version of the generic mapping tools. Eos, Transactions American Geophysical Union, 76(33), 329-329.

Zumberge, J. F., Heflin, M. B., Jefferson, D. C., Watkins, M. M., \& Webb, F. H. (1997). Precise point positioning for the efficient and robust analysis of GPS data from large networks. Journal of geophysical research: solid earth, 102(B3), 5005-5017.

URL-1: https://www.spaceweatherlive.com/en/archive, (Erişim Tarihi: 12 Mart 2017).

URL-2: https://gipsy-oasis.jpl.nasa.gov/gipsy/docs/GD2P_PPP.pdf, (Erişim Tarihi: 12 Mart 2017). 